\title{
Size Effects on Tensile Strength of Lotus-Type Porous Copper
}

\author{
Hidekazu Sueno, Masakazu Tane*, Jae-Soung Park, Soong-Keun Hyun and Hideo Nakajima \\ The Institute of Scientific and Industrial Research, Osaka University, Ibaraki 567-0047, Japan \\ Effects of specimen thickness on the tensile strength of lotus-type porous copper were investigated. The ultimate tensile strength in the \\ direction perpendicular to the longitudinal axis of pores hardly depends on the thickness of a specimen when the width of the specimen is large \\ enough compared with the pore diameter, while the ultimate tensile strength increases with an increase in the thickness when the width is not \\ large enough compared with the pore diameter. The $0.2 \%$ offset strength in the direction and strain at the peak stress depend on the thickness of \\ specimens; the $0.2 \%$ offset strength decreases with an increase in the thickness, and the strain at peak stress increases with the increase in the \\ thickness. [doi:10.2320/matertrans.47.2203]
}

(Received February 28, 2006; Accepted May 15, 2006; Published September 15, 2006)

Keywords: lotus-type porous metals, copper, size effect, tensile strength

\section{Introduction}

Porous metals such as metallic foams possess randomly dispersed nonuniform pores of high porosity. ${ }^{1)}$ These porous metals are used as impact-energy absorbers or the core of sandwich panels because their strength is considerably low. Recently, lotus-type porous metals (lotus metals), possessing cylindrical pores aligned in one direction, attract much attention. ${ }^{2-5)}$ This is because the mechanical properties of lotus metals are superior to those of the conventional porous metals owing to their unique porous structure. Thus, lotus metals are promising not only as functional materials but also as structural materials. In order to use lotus metals as structural materials, it is essential to clarify their mechanical properties in detail.

The mechanical properties of lotus metals have been studied, and the effects of porosity and anisotropic porous structure have been revealed; the ultimate tensile strength ${ }^{6}$ and Young's modulus ${ }^{7)}$ in the direction parallel to the longitudinal axis of pores decrease almost linearly with an increase in porosity, while those in the direction perpendicular to the longitudinal axis to the pores rapidly decrease with the increase in porosity. Here, it is to be noted that the mechanical properties of porous metals probably depend on their specimen dimensions when the dimensions are not large enough compared with the pore size; ${ }^{8,9)}$ the strength decreases with decrease in specimen dimensions. This is because the strength of the surface part of porous specimens is weak compared with that of the inner part, and the effect of the weak surface part increases with a decrease in specimen dimensions; when specimen dimensions are large enough, the effect of weak surface part is negligible. Consequently, to obtain the reliable mechanical strength of porous metals, it is important to clarify the influence of specimen dimensions on the mechanical strength and to use specimens with sufficient dimensions for mechanical testings.

In the present study, we studied the effects of specimen dimensions on the tensile properties of lotus-type porous copper (lotus copper). Tensile tests were carried out on lotus copper specimens with three kinds of thickness, and the effects of the thickness on tensile properties were clarified.

*Corresponding author, E-mail: mtane@sanken.osaka-u.ac.jp
Table 1 Total pressure $\left(P_{\mathrm{T}}\right)$, and partial pressure of argon and hydrogen $\left(P_{\mathrm{Ar}}\right.$ and $\left.P_{\mathrm{H}_{2}}\right)$ for the preparation of ingots (Sample No. N1, N2 and A-E); porosity $(p)$, average pore diameter $(d)$ and coefficient of variation of pore diameter $(\mathrm{Cv})$ for the prepared ingots.

\begin{tabular}{|c|c|c|c|c|c|c|}
\hline \multirow{2}{*}{$\begin{array}{c}\text { Sample } \\
\text { No. }\end{array}$} & \multirow{2}{*}{$\begin{array}{c}\text { Total pressure } \\
P_{\mathrm{T}} / \mathrm{MPa}\end{array}$} & \multicolumn{2}{|c|}{ Partial pressure } & \multirow{2}{*}{$\begin{array}{c}\text { Porosity } \\
p(\%)\end{array}$} & \multirow{2}{*}{$\begin{array}{c}d \\
/ \mu \mathrm{m}\end{array}$} & \multirow{2}{*}{$\begin{array}{l}\mathrm{Cv} \\
(\%)\end{array}$} \\
\hline & & $P_{\mathrm{Ar}} / \mathrm{MPa}$ & $P_{\mathrm{H}_{2}} / \mathrm{MPa}$ & & & \\
\hline N1 & 0.40 & 0.40 & 0 & 0 & - & - \\
\hline $\mathrm{N} 2$ & 0.40 & 0.40 & 0 & 0 & - & - \\
\hline $\mathrm{A}$ & 0.30 & 0 & 0.30 & 33.3 & 247.5 & 39.2 \\
\hline B & 0.10 & 0 & 0.10 & 43.8 & 107.4 & 38.2 \\
\hline $\mathrm{C}$ & 0.40 & 0.10 & 0.30 & 50.0 & 238.2 & 38.4 \\
\hline D & 0.40 & 0 & 0.40 & 54.9 & 292.4 & 47.9 \\
\hline $\mathrm{E}$ & 0.10 & 0 & 0.15 & 65.8 & 719.0 & 62.3 \\
\hline
\end{tabular}

\section{Experimental Procedure}

Five kinds of lotus copper ingots (samples A, B, C, D and E) were prepared through the unidirectional solidification in pressurized hydrogen and argon atmospheres. ${ }^{2)}$ Furthermore, two nonporous ingots (samples N1 and N2) were prepared through the unidirectional solidification in a pressurized argon atmosphere. Table 1 shows the total pressure of the mixed gas and the partial pressure of the hydrogen and argon gas for the preparation of the ingots. Figure 1 shows the cross section of each sample, where the cross section is perpendicular to the longitudinal axis of pores. Porosity $p$ of each sample, shown in Table 1, was determined from the relative density using the equation:

$$
p=1-\frac{\rho}{\rho_{0}},
$$

where $\rho_{0}$ is the density of a nonporous sample and $\rho$ is the relative density of a porous sample. The pore diameter of each sample was measured with an image analyzer (WinROOF, Mitani Corp.); the averages $(d)$ and coefficient of variations $(\mathrm{Cv})$ of pore diameter are shown in Table 1.

Tensile specimens (Fig. 2), whose longitudinal directions are perpendicular to the longitudinal axis of pores, were cut out from each ingot by using a spark-erosion wire cutting machine; for all tensile specimens, the gage length and width $w$ are 20 and $6.7 \mathrm{~mm}$, respectively; Table 2 shows the ratio 
(a)

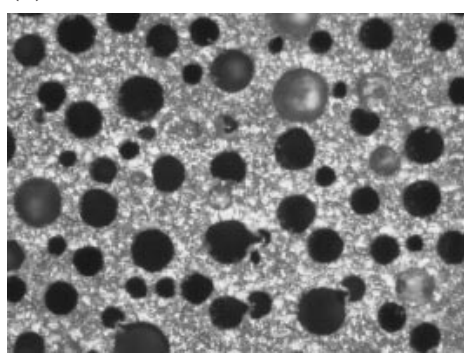

(d)

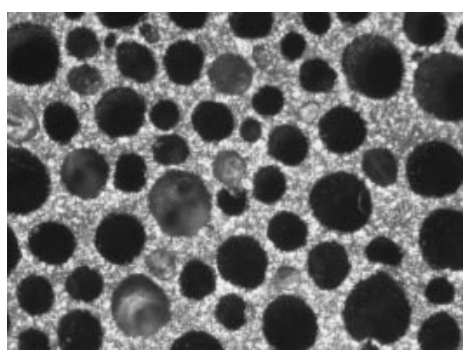

(b)

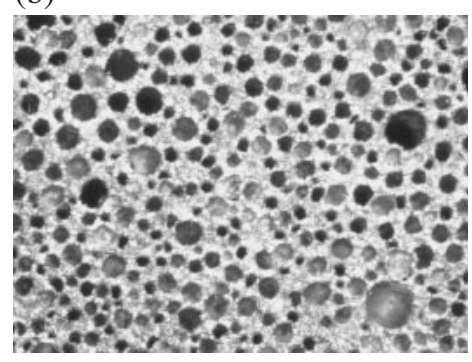

(e)

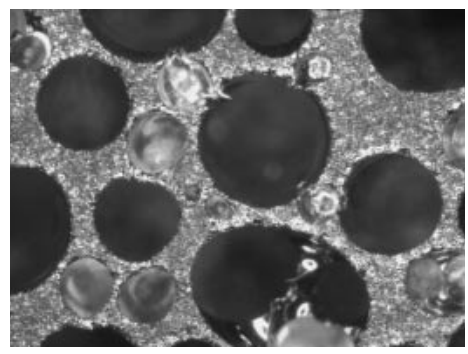

(c)

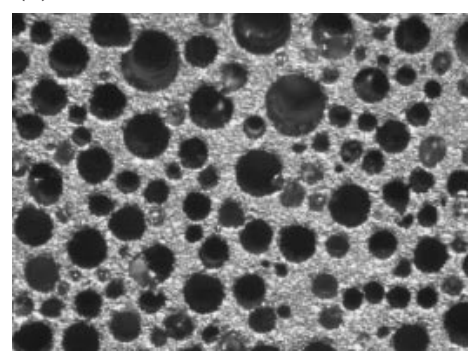

$500 \mu \mathrm{m}$

Fig. 1 Microstructures of cross sections of lotus copper ingots; the cross section is perpendicular to the longitudinal axis of pores: (a) sample A, (b) sample B, (c) sample C, (d) sample D and (e) sample E.

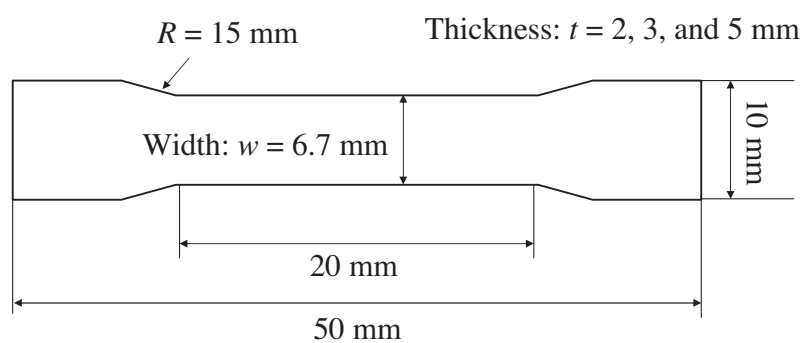

Fig. 2 Schematic illustration showing the dimensions of a tensile specimen.

of the width $w$ to average pore diameter $d, w / d$. For each ingot, tensile specimens with the thickness of 2, 3 and $5 \mathrm{~mm}$ were prepared. Table 2 shows the average and coefficient of variation of porosities among specimens with the same thickness. Furthermore, tensile specimens, whose longitudinal directions are parallel and perpendicular to the solidification direction, are cut out from $\mathrm{N} 1$ and $\mathrm{N} 2$ samples, respectively.

Uniaxial tensile tests were carried out on prepared specimens using an Instron Universal Testing Machine (Model 4482, Instron Corp., Carton, MA, USA) at room temperature; the crosshead speed was $1 \mathrm{~mm} / \mathrm{min}$.

\section{Experimental Results}

Figure 3 shows the stress-strain curves of nonporous specimens in the directions (a) parallel (sample N1) and (b) perpendicular (sample N2) to the solidification direction; the thicknesses of specimens are 2, 3 and $5 \mathrm{~mm}$. The strain at a peak stress (ultimate tensile strength) clearly depends on the thickness of a specimen; it increases with an increase in the thickness. The ultimate tensile strength hardly depends on the
Table 2 Thickness $t$ and number $N$ of specimens cut out from sample A-E; the averages (AP) and coefficient of variations (CVP) of porosity for the specimens with each thickness; the ratio of the width of specimen $w$ to averade pore diameter $d, w / d$.

\begin{tabular}{|c|c|c|c|c|c|}
\hline Sample No. & $t / \mathrm{mm}$ & $N$ & AP $\%$ & CVP \% & $w / d$ \\
\hline \multirow{3}{*}{ N1 } & 2 & 4 & 0.0 & - & - \\
\hline & 3 & 5 & 0.0 & - & - \\
\hline & 5 & 5 & 0.0 & - & - \\
\hline \multirow{3}{*}{ N2 } & 2 & 10 & 0.0 & - & - \\
\hline & 3 & 10 & 0.0 & - & - \\
\hline & 5 & 10 & 0.0 & - & - \\
\hline \multirow{3}{*}{ A } & 2 & 10 & 34.3 & 4.75 & 27.1 \\
\hline & 3 & 10 & 33.1 & 4.33 & 27.1 \\
\hline & 5 & 10 & 32.6 & 5.81 & 27.1 \\
\hline \multirow{3}{*}{ B } & 2 & 10 & 44.5 & 0.77 & 62.4 \\
\hline & 3 & 10 & 43.8 & 1.22 & 62.4 \\
\hline & 5 & 10 & 43.2 & 1.33 & 62.4 \\
\hline \multirow{3}{*}{$\mathrm{C}$} & 2 & 10 & 50.6 & 1.43 & 28.1 \\
\hline & 3 & 10 & 49.7 & 0.86 & 28.1 \\
\hline & 5 & 10 & 49.6 & 0.48 & 28.1 \\
\hline \multirow{3}{*}{$\mathrm{D}$} & 2 & 10 & 55.5 & 0.68 & 22.9 \\
\hline & 3 & 10 & 54.8 & 0.89 & 22.9 \\
\hline & 5 & 10 & 54.4 & 0.78 & 22.9 \\
\hline \multirow{3}{*}{$\mathrm{E}$} & 2 & 10 & 66.3 & 2.19 & 9.3 \\
\hline & 3 & 10 & 65.9 & 1.43 & 9.3 \\
\hline & 5 & 10 & 65.1 & 1.80 & 9.3 \\
\hline
\end{tabular}

thickness, while $0.2 \%$ offset strength decreases with an increase in thickness.

Figure 4(a) shows the stress-strain curves of specimens cut out from sample $\mathrm{B}$, where the thicknesses of the specimens are 2, 3 and $5 \mathrm{~mm}$. Size effects on the stress-strain behavior are consistent with those on the stress-train behavior of 
(a)

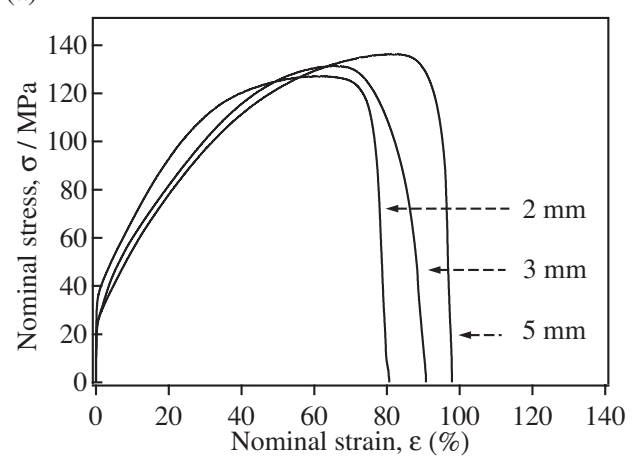

(b)

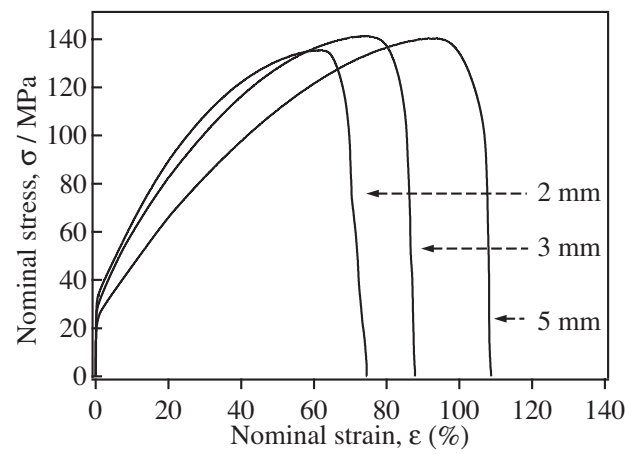

Fig. 3 The stress-strain curves of nonporous copper specimens fabricated through unidirectional solidification, where the thicknesses of the specimens are 2, 3 and $5 \mathrm{~mm}$ : loading direction is (a) parallel (sample N1) and (b) perpendicular (sample N2) to the solidification direction.

(a)

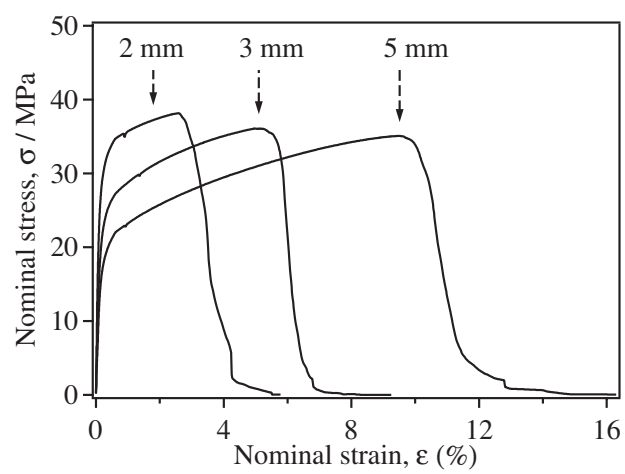

(b)

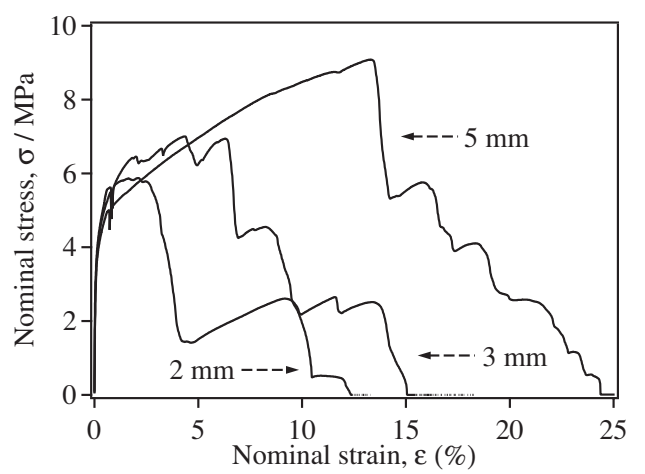

Fig. 4 The stress-strain curves of lotus copper specimens in the direction perpendicular to the longitudinal axis of pores: (a) specimens with the thickness of 2, 3 and $5 \mathrm{~mm}$ were cut out from sample B and (b) specimens with the thickness of 2, 3 and $5 \mathrm{~mm}$ were cut out from sample E.

nonporous specimens: the strain at a peak stress increases with an increase in the thickness of specimens; tensile strength hardly depends on the thickness; $0.2 \%$ offset strength decreases with the increase in the thickness. These size effects are common to samples A, B, C and D, which possess the large values of $w / d$ (for the values of $w / d$, see Table 2).

Figure 4(b) shows the stress-strain curves of specimens cut out from sample $\mathrm{E}$ with the small value of $w / d$, where the thicknesses of the specimens are 2, 3 and $5 \mathrm{~mm}$. The stressstrain behaviors are different from those of sample B; the stress-strain curves of the specimens cut out from sample E are very rough. The ultimate tensile strength increases with an increase in specimen thickness, and $0.2 \%$ offset strength slightly depends on the thickness.

Figure 5 summarizes the effect of specimen thickness $t$ on the $0.2 \%$ offset strength of nonporous and lotus copper specimens; for each sample (ingot), the average of $0.2 \%$ offset strength of specimens with a thickness is normalized with the average of specimens with the thickness of $5 \mathrm{~mm}$. For all samples, the (normalized) $0.2 \%$ offset strength decreases with an increase in the thickness $t$. The dependence of $0.2 \%$ offset strength on the thickness is different among samples.

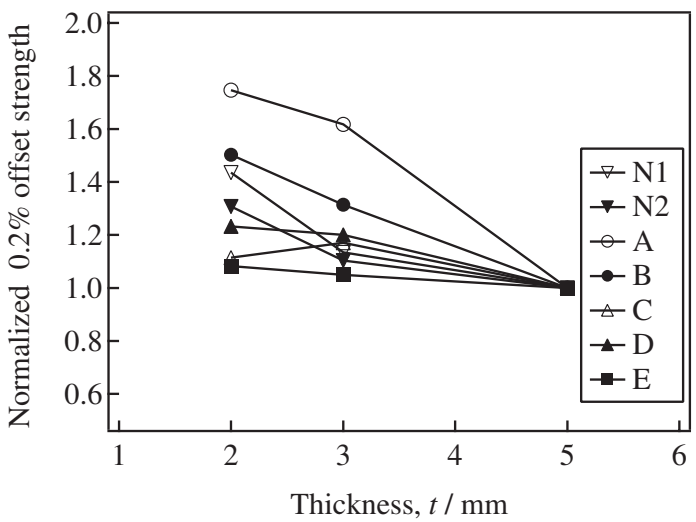

Fig. 5 Normalized $0.2 \%$ offset strength of nonporous and lotus copper specimens as a function of specimen thickness $t$; for each sample, the average of $0.2 \%$ offset strength of specimens with a thickness is normalized with the average strength of specimens with the thickness of $5 \mathrm{~mm}$.

Figure 6 summarizes the effect of specimen thickness $t$ on the ultimate tensile strength of nonporous and lotus copper specimens; for each sample, the average ultimate tensile strength of specimens with a thickness is normalized with 


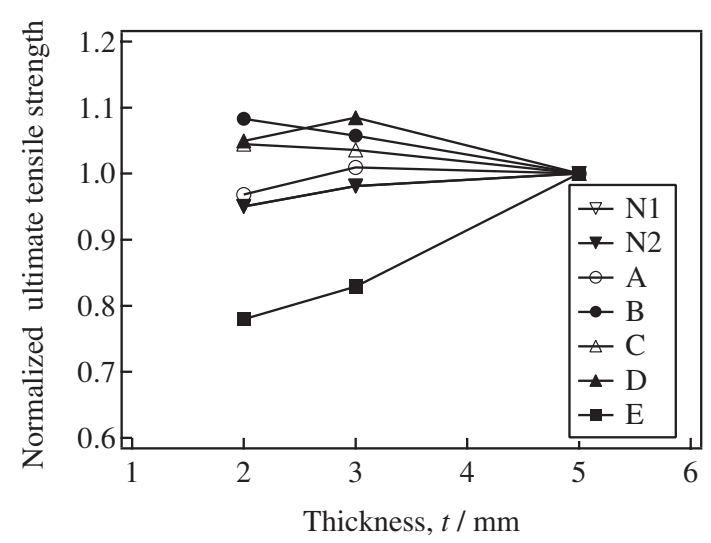

Fig. 6 Normalized ultimate tensile strength of nonporous and lotus copper specimens as a function of specimen thickness $t$; for each sample, the average of ultimate tensile strength of specimens with a thickness is normalized with the average strength of specimens with the thickness of $5 \mathrm{~mm}$.

the average of specimens with the thickness of $5 \mathrm{~mm}$. For specimens with the large value of $w / d$ (specimens cut out from samples A, B, C and D) and nonporous specimens (specimens cut out from samples N1 and N2), the (normalized) ultimate tensile strength hardly depends on the thickness. On the other hand, for specimens with the small value of $w / d$ (specimens cut out from sample E), the (normalized) ultimate tensile strength increases with an increase in the thickness.

\section{Discussion}

In general, for tensile test on bulk metals, the shape of a specimen has an influence on the local elongation (strain after a peak stress); uniform elongation hardly depends on the specimen shape, while local elongation depends on specimen shape (the ratio of gage length to the square root of gage section). ${ }^{10)}$ However, specimen shape has little influence on the ultimate tensile strength, yield strength and strain at peak stress. ${ }^{10)}$ In the present study, the dependence of $0.2 \%$ offset strength and the strain at peak stress on the thickness of specimens is observed for nonporous and porous specimens. It is speculated that this dependence of $0.2 \%$ offset strength and strain at peak stress on specimen thickness originates in the size effect on strain, and the size effect originates in nonuniform deformation. The microstructures of nonporous copper and matrix of lotus copper are different from those of fine-grained polycrystalline copper; the fiber texture of large grain size $(1-2 \mathrm{~mm})$, whose preferential growth direction is [001] direction, ${ }^{7)}$ exist in lotus copper matrix and nonporous copper. Because of this anomalous microstructure, nonuniform deformation occurs even when the strain is small. This nonuniform deformation possibly causes the size effects on strain, i.e., measured strain depends on specimen thickness. Therefore, $0.2 \%$ offset strength (stress at $0.2 \%$ strain) and strain at peak stress depend on the thickness owing to the dependence of strain on specimen thickness.

Size effect on ultimate tensile strength is related to the strength of the surface part of porous metals. The strength of the surface part is weak compared with that of the inner part, (a)

(b)

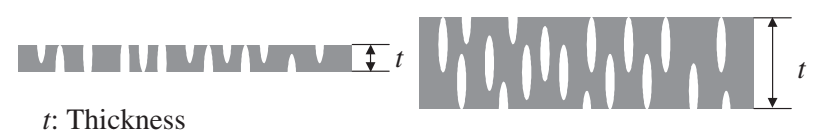

Fig. 7 Schematic illustration showing lotus copper specimens with (a) small and (b) large thicknesses; longitudinal direction of pores is parallel to the thickness direction.

and the effect of weak surface part increases with a decrease in specimen dimensions. When specimen dimensions are not large enough compared with pore diameter, the strength of weak surface part is dominant for the strength of porous metals. Therefore, for specimens cut out from sample E (specimens with the largest value of $w / d$ ), the ultimate tensile strength is weak and the stress-strain curve is rough, because the effect of the surface part is large. Furthermore, for sample E, the ultimate strength increases with an increase in specimen thickness. This is because the strength of the surface part, which depends on the morphology of the part, increases with the increase in thickness. Here, we consider lotus copper specimens, whose longitudinal direction of pores is parallel to the thickness direction (Fig. 7). When the thickness is small, most pores are not surrounded by a metal matrix in the thickness direction. When the thickness is large, the fraction of pores, surrounded by the metal matrix, increases. This increase of fraction of pores surrounded by the metal matrix provides the enhancement of the strength of the surface part.

\section{Conclusions}

We carried out the tensile test on lotus copper specimens with various thicknesses in the direction perpendicular to the longitudinal axis of pores, and clarified the effects of specimen thickness on the ultimate tensile strength, $0.2 \%$ offset strength and strain at peak stress. The following conclusions were obtained:

(i) The ultimate tensile strength is independent of the thickness of specimens when the width of specimen is large enough compared with pore diameter. While the ultimate tensile strength increases with an increase in the thickness when the width of the specimen is not large enough.

(ii) The $0.2 \%$ offset strength decreases with an increase in the thickness of specimen, and strain at peak stress increases with the increase in specimen thickness. These size effects are probably caused by the dependence of strain on the thickness, which originates in the nonuniform deformation of the metal matrix.

\section{Acknowledgement}

This work was also supported by "Priority Assistance for the Formation of Worldwide Renowned Centers of Research for the 21st Century COE Program (Project: Center of Excellence for Advanced Structural and Functional Materials Design)" from the Ministry of Education, Culture, Sports, Science and Technology of Japan. 


\section{REFERENCES}

1) L. J. Gibson and M. F. Ashby: Cellular Solids, 2nd ed. (Cambridge University Press, UK, 1997).

2) H. Nakajima, S. K. Hyun, K. Ohashi, K. Ota and K. Murakami: Colloids Surfaces A 179 (2001) 209-214.

3) H. Nakajima, T. Ikeda and S. K. Hyun: Adv. Eng. Mater. 6 (2004) $377-$ 384

4) T. Ikeda, T. Aoki and H. Nakajima: Metall. Trans. A 36A (2005) 77-

5) V. Shapovalov and L. Boyko: Avd. Eng. Mater. 6 (2004) 406-410.
6) S. K. Hyun, K. Murakami and H. Nakajima: Mater. Sci. Eng. A 299 (2001) 241-248.

7) T. Ichitsubo, M. Tane, H. Ogi, M. Hirao, T. Ikeda and H. Nakajima: Acta Mater. 50 (2002) 4105-4115.

8) P. R. Onck, E. W. Andrews and L. J. Gibson: Int. J. Mech. Sci. 43 (2001) 681-699.

9) E. W. Andrews, G. Gioux, P. Onck and L. J. Gibson: Int. J. Mech. Sci. 43 (2001) 701-713.

10) H. Nakazawa: Test Manual of Metallic Materials, Japan Standard Association (1987) pp. 51-53. 